\title{
Near-perfect modulator for polarization state of light
}

\author{
Yi-Jun Jen, Yung-Hsun Chen, Ching-Wei Yu, and Yen-Pu Li \\ Department of Electro-Optical Engineering, National Taipei University of Technology, No. 1, \\ Sec. 3, Chung-Hsiao E. Rd. Taipei, Taiwan 106 \\ jyjun@ntut.edu.tw
}

\begin{abstract}
The ability of a weakly anisotropic thin film to modulate the polarization state of light was considered. We found that, in a configuration for enhanced polarization conversion, most polarization states can be generated by tuning the direction of incident linear polarization and the aspect of the anisotropic thin film. The modulation for omniform polarization state can be extended to a broad wavelength range by adding an isotropic thin film in the system.
\end{abstract}

Keywords: thin films, optical properties, birefringence, polarization-selective devices, modulators.

\section{INTRODUCTION}

The polarization state is an important characteristic of an electromagnetic plane wave. Recent studies [1-3] have demonstrated that a method that integrates the interference effect and the retardation effect can enhance a change in polarization state by introducing light at obliquely incidence onto a columnar thin film [4] that is optically birefringent. Under the condition that the angles of incidence and reflection remain the same, the polarization state of light reflected from an anisotropic $\mathrm{MgF}_{2}$ thin film can be modulated from a linear polarization state to a circular polarization state merely by rotating the thin film about its normal. The transmission direction of the linear polarizer in the incident optical path is tunable in addition to the rotatable film. It was found that most polarization states characterized by the ellipticity and the azimuth angle of an elliptical polarization can be modulated by the two-dimensional tunable orientations of the polarizer and anisotropic thin film. The modulation for polarization state can be extended in a broad wavelength range by adding an isotropic thin film in the system.

In conventional optics, the general method for obtaining a particular polarization state is to use a phase retarder (which is also called a wave plate). The different optical paths of the components of the electric field change the polarization state of a linear polarized beam that propagates through the retarder. The optical path difference (difference between refractive indices multiplied by geometrical thickness) must be sufficiently high to change the polarization state. For a quarter wave plate, the relative optical path-length difference should be an odd multiple of a quarter wavelength to modulate linear polarization to circular polarization. The wave plate can be an electro-optical material or a liquid crystal whose optical path shift is controlled by an external electric field. Another way to modulate the polarization state, introduced here, involves a weak anisotropic optical thin film that is columnar and can be easily fabricated by tilting the normal of the substrate at an angle with respect to the deposition flux; this method is called the oblique angle deposition technique $[5,6]$.

In a single-layered system, the polarization state of the light that is reflected from the film can be converted from that of the light that is obliquely incident on a birefringent thin film between a dense incident medium and a rare substrate medium. Three mixed mechanisms [1], including phase retardation, interference and total reflection effects in the anisotropic thin film, enhance the polarization conversion that is observed in a certain range of the angular 
spectrum. The degree of polarization conversion can be tuned by rotating the film with respect to its surface normal.

An initial study has demonstrated that polarization can be changed from linear to circular states by rotating the film. Since any polarization state can be specified by the shape and orientation of an elliptical polarization state, given by the ellipticity and azimuth angle, two input parameters must be introduced to modulate the polarization state. In this letter, the direction of the linear oscillating electric field of the incident beam and the aspect of the film become the two tunable input parameters that are used to modulate the polarization state of the reflected beam.

\section{SIMULATION OF MODULATION ABILITY FOR A SINGLE ANISOTROPIC THIN FILM}

\subsection{The single-layered configuration}

The proposed single-layered configuration [1] for converting the polarization state is employed here to investigate the possible polarization state that can be obtained by tuning the input parameters under the prerequisite that the optical paths of the incident beam and reflected beam remain unchanged. Figure 1 presents the configuration. The typical birefringent thin film is a $\mathrm{MgF}_{2}$ columnar film with thickness $\mathrm{d}=1100 \mathrm{~nm}$, and the aspect of the rotatable film is described by the angle $\delta$ between the deposition plane and the plane of incidence. The columnar tilt angle is $\beta=41.5^{\circ}$. The columnar array is effectively an optically birefringent continuum one of whose principal axes is in the columnar growth direction. Accordingly, the principal axes $(1,2,3)$ are defined as in Fig. 1 and the three principal indices of a typical $\mathrm{MgF}_{2}$ thin film are $n_{1}=1.304, n_{2}=1.303$ and $n_{3}=1.323$ at the wavelength of $632.8 \mathrm{~nm}$. The medium of incidence and reflection is dielectric with index of refraction 1.515, and the substrate medium is air. The direction of the incident oscillating electric field is given by the angle $\xi$ between the transmission direction of the polarizer and the parallel polarization direction.

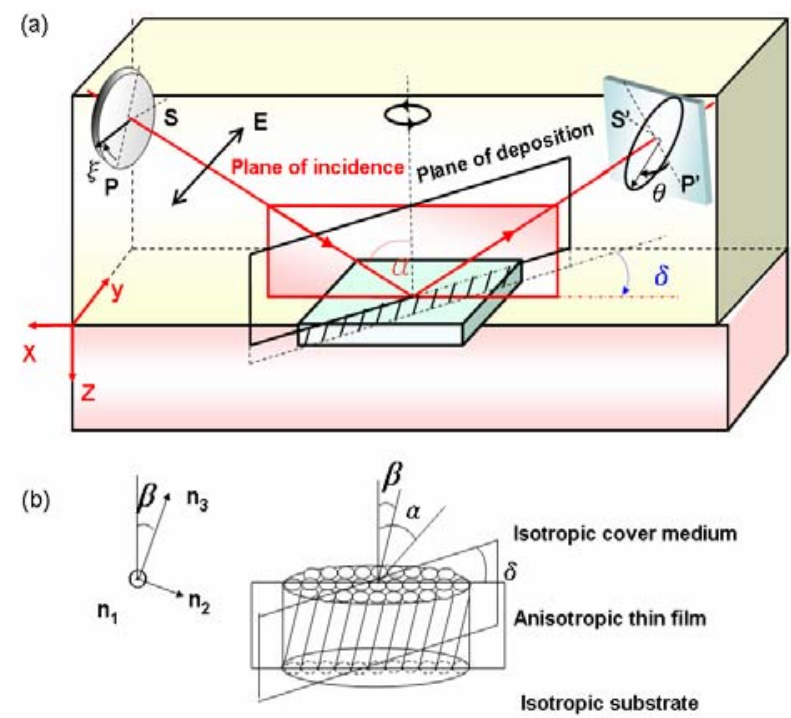

Fig. 1. Single-layered configuration for generating enhanced polarization conversion. Principal axes $(1,2,3)$ and laboratory axes $(\mathrm{x}, \mathrm{y}, \mathrm{z})$ are shown. The plane of incidence is the $y-z$ plane. 


\subsection{The modulated elliptical polarization}

The reflected polarization state is characterized by an ellipticity and an azimuth angle [7]. The ellipticity is defined as the ratio of the electric strength of the semi-minor axis to that of the semi-major axis of the ellipse. The positive and negative values of ellipticity correspond to right-handed and left-handed polarization states, respectively. The azimuth angle is defined as the angle between the major axis of the ellipse and the direction of the reflected p-polarized beam.

The degree of conversion of the polarization state increases with the angle $\delta$ from $0^{\circ}$ to $90^{\circ}$. For a pure p-polarized beam, $90.962 \%$ of the incident p-polarized light is converted to reflected s-polarized light at angle of incidence $\alpha=57.52^{\circ}$ at $\delta=90^{\circ}$. The relationship between the tunable parameters $(\xi, \delta)$ and the elliptical parameters of modulated polarization $(e, \theta)$ is determined theoretically based on Berreman's calculus [6]. That relationship between the electric field of the reflected light $\left(E_{s}^{r}, E_{p}^{r}\right)$ can be derived from the incident light $\left(E_{s}^{i}, E_{p}^{i}\right)$ :

$$
\left[\begin{array}{c}
E_{s}^{r} \\
E_{p}^{r}
\end{array}\right]=\left[\begin{array}{cc}
r_{s s} & r_{p s} \\
r_{s p} & r_{p p}
\end{array}\right]\left[\begin{array}{l}
E_{s}^{i} \\
E_{p}^{i}
\end{array}\right]=\left[\begin{array}{cc}
r_{s s}(\delta) & r_{p s}(\delta) \\
r_{s p}(\delta) & r_{p p}(\delta)
\end{array}\right]\left[\begin{array}{c}
E^{i} \sin \xi \\
E^{i} \cos \xi
\end{array}\right],
$$

where $r_{i j}$ is the coefficient of reflection defined as a ratio of the $j$-polarized reflected electric field to the $i$-polarized incident electric field $(i, j=s, p)$. Those reflection coefficients can be derived using Berreman's calculus. When the optical constants of the anisotropic thin film are fixed, the only variable in the transfer matrix is $\delta$. The following equation describes the relationship between the output elliptical parameters $(e, \theta)$ and the reflected electric field $\left(E_{s}^{r}(\delta, \xi), E_{p}^{r}(\delta, \xi)\right)[7]$ :

$$
\frac{E_{s}^{r}(\delta, \xi)}{E_{p}^{r}(\delta, \xi)}=\frac{\tan \theta+i e}{1-i e \tan \theta}
$$

where the tunable parameters $\delta$ and $\xi$ are in the ranges $0 \leq \delta<2 \pi$ and $0 \leq \xi<\pi$ respectively. Any polarization state must be within the ranges $-1 \leq e \leq 1$ and $-\pi / 2 \leq \theta<\pi / 2$.

\subsection{Possible modulated range of polarization state}

For a pure s-polarized incident light, the polarization state of the reflected light can be altered from linear to nearly circular by merely rotating the film from $\delta=0^{\circ}$ to $\delta=90^{\circ}$ at the angle of incidence $\alpha=53.4^{\circ}$. Therefore the angle of incidence $\alpha=53.4^{\circ}$ was adopted to investigate the largest range of modulation by tuning the parameters $(\xi, \delta)$. In the calculation, the possible values $(\xi, \delta)$ that yield a specific ellipticity $e$ within the range $e \pm 0.004$ were used to calculate the azimuth angles, and the area between the maximum and minimum $\theta$ values is marked as a rectangular region in $(e, \theta)$ coordinates. All possible modulated polarization states are marked in $(\varepsilon=\arctan e, \theta)$ coordinates and Poincaré sphere [7], as presented in Video 1. 
(a)

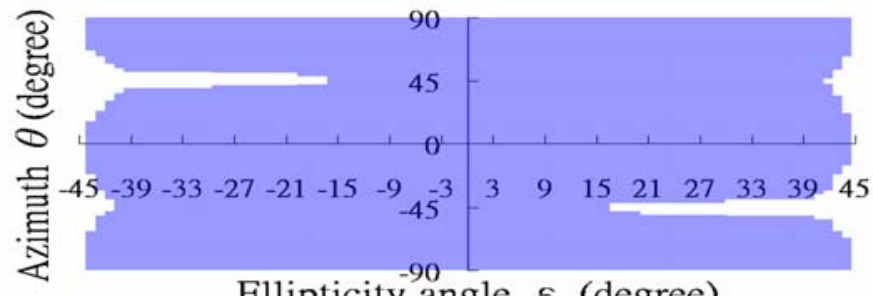

Ellipticity angle $\varepsilon$ (degree)

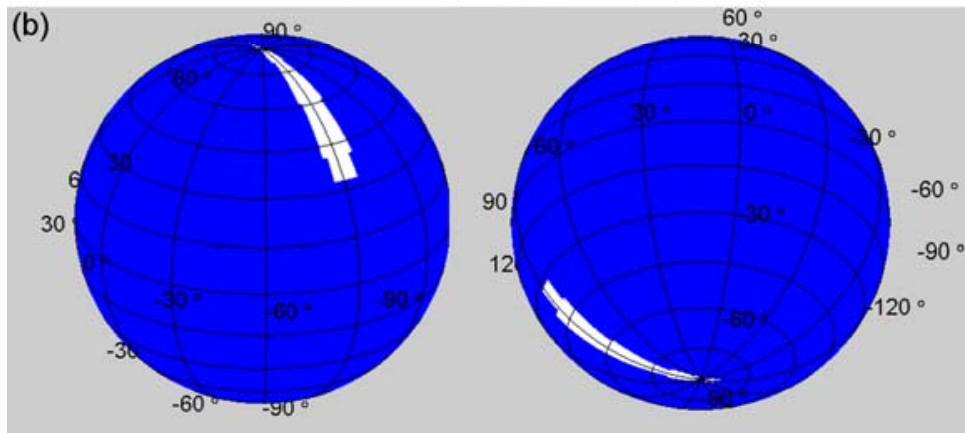

Video 1. All possible modulated polarization states in (a) $(\varepsilon, \theta)$ coordinates. Poincaré sphere (North Pole view and South Pole view) [QuickTime, 2.8 MB].

The latitude and longitude of the Poincare sphere are associated with the azimuth $\theta$ and ellipticity angle $\varepsilon$ of a polarization state, respectively. The longitude is double the azimuth $\theta$ and the latitude is double the ellipticity angle $\varepsilon$. There is $97.686 \%$ area on the Poincare sphere is marked as the possible modulated range that can be generated by rotating the film and polarizer.

\section{EXPERIMENTAL MODULATION BY AN ANISOTROPIC MgF $_{2}$ THIN FILM}

One example of the modulation was implemented experimentally. The aforementioned $\mathrm{MgF}_{2}$ thin film was deposited on a BK7 substrate with thermal evaporation. The normal of the substrate was tilted at an angle $70^{\circ}$ with respect to the deposition flux. The deposition rate was maintained at $0.6 \mathrm{~nm} / \mathrm{s}$ read by quartz thickness monitor next to the substrate during deposition. The optical constants of the anisotropic thin film were found to be $\left(n_{1}, n_{2}\right.$, $\left.n_{3}, \beta\right)=\left(1.304,1.303,1.323,41.5^{\circ}\right)$ by measuring the angular spectrum of polarization conversion reflectance [8]. The thickness of the film was $1100 \mathrm{~nm}$.

The substrate was attached to a BK7 prism to form a BK7 prism/anisotropic $\mathrm{MgF}_{2}$ film/ air system and the thin-film plate was rotatable in modulation. The ellipticity of the reflected beam can be varied from 0.028 to 0.973 by rotating the film from $\delta=58^{\circ}$ to $\delta=265^{\circ}$. During the rotation, the polarizer was adjusted in the range from $\xi=140^{\circ}$ and $\xi=95^{\circ}$ to maintain the azimuth angle at $\theta=46^{\circ} \pm 2^{\circ}$, as shown in Fig. 2(a). Figure 2(b) shows another interesting result: provided the film remains fixed at $\delta=90^{\circ}$, the ellipticity can be modulated from 0.018 to 0.955 while the azimuth angle remains within the range $\theta=-42^{\circ} \pm 2^{\circ}$, by tuning the polarizer from $\xi=42^{\circ}$ to $\xi=86^{\circ}$. 

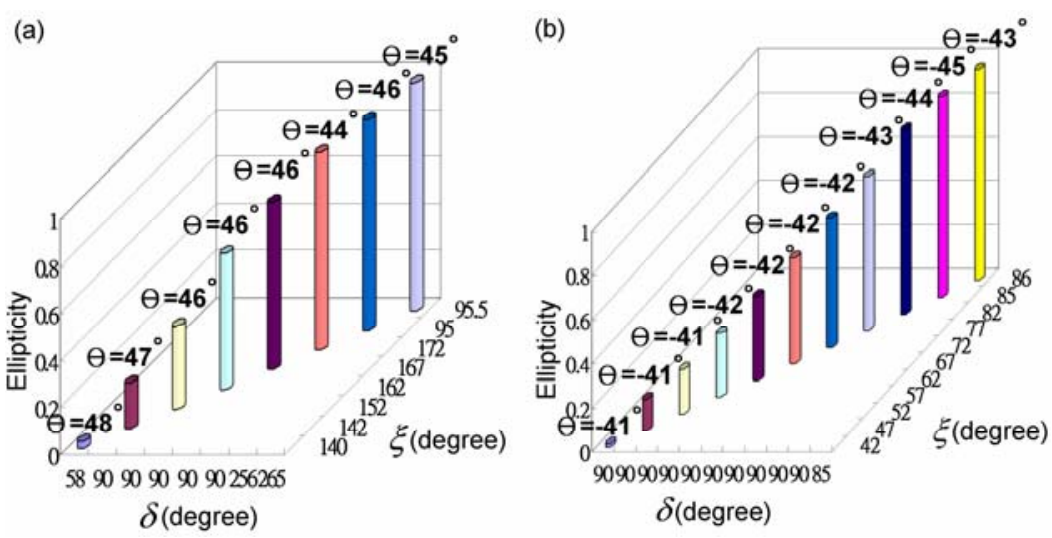

Fig. 2. (a) Modulation of ellipticity from 0.028 to 0.973 by tuning $(\xi, \delta)$, when the azimuth angle is within the range $\theta=46^{\circ} \pm 2^{\circ}$. (b) Modulation of ellipticity from 0.018 to 0.955 by tuning ( $\xi$ $, \delta)$, when the azimuth angle is within the range $\theta=-42^{\circ} \pm 2^{\circ}$.

\section{SIMULATION OF MODULATION ABILITY FOR A TWO-LAYERED CONFIGURATION}

A two-layered configuration (BK7 prism/isotropic $\mathrm{MgF}_{2}$ film/anisotropic $\mathrm{MgF}_{2}$ film/ air) that has been proposed to cause a broadband polarization conversion was also studied to determine its effectiveness in modulating polarization states. All optical constants of the configuration at wavelength $632.8 \mathrm{~nm}$ were used and dispersion was ignored. The index of refraction of the isotropic $\mathrm{MgF}_{2}$ film of thickness $256 \mathrm{~nm}$ is 1.362 and the principal indices of the anisotropic $\mathrm{MgF}_{2}$ thin film with thickness $1450 \mathrm{~nm}$ are $\left(n_{1}, n_{2}, n_{3}, \beta\right)=(1.313,1.312$, $\left.1.327,39.0^{\circ}\right)$. The angle of incidence is fixed at $59.55^{\circ}$. When the orientations of the film and the polarizer become $(\xi, \delta)=\left(0^{\circ}, 90^{\circ}\right)$, the ellipticity of the reflected beam remains high, at over 0.97, for wavelengths from $470 \mathrm{~nm}$ to $600 \mathrm{~nm}$, as shown in Fig. 3. Based on the above method, possible reflected polarization states that are modulated by tuning the parameters $(\xi, \delta)$ were simulated for wavelengths $520 \mathrm{~nm}, 540 \mathrm{~nm}, 560 \mathrm{~nm}, 580 \mathrm{~nm}$ and $600 \mathrm{~nm}$. Table 1 presents the percentage of the possible modulated range on the Poincare sphere. An average $99.898 \%$ of the polarization state can be modulated for wavelengths from $520 \mathrm{~nm}$ to $600 \mathrm{~nm}$.

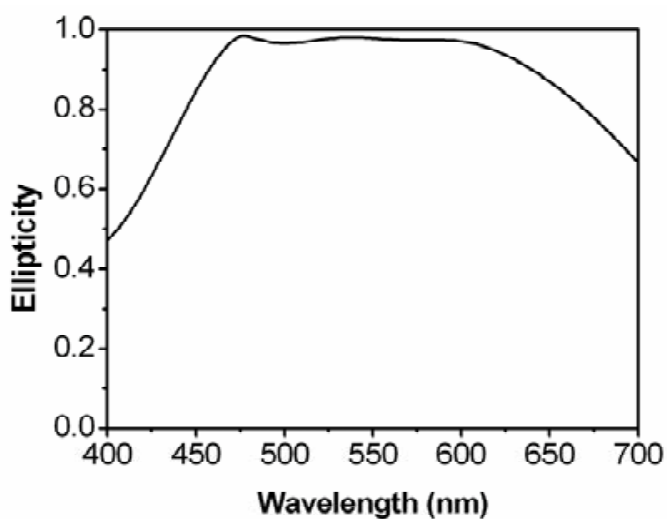

Fig. 3. The ellipticity of the reflected beam in the $\mathrm{BK} 7$ prism/isotropic $\mathrm{MgF}_{2}$ film/anisotropic $\mathrm{MgF}_{2}$ film/air system. 
Table 1. The percentage of the possible modulated range on the Poincaré sphere.

\begin{tabular}{llllll}
\hline Wavelength $(\mathrm{nm})$ & 520 & 540 & 560 & 580 & 600 \\
\hline Possible modulated range (\%) & 99.527 & 99.995 & 99.992 & 99.992 & 99.989 \\
\hline
\end{tabular}

\section{CONCLUSION}

We have demonstrated that an anisotropic thin film fabricated by oblique angle deposition technique can be applied to obtain most polarization states. Tuning the orientation of the polarizer for obliquely incident light and the aspect of the deposition plane of the film can yield over $99 \%$ of polarization state at a fixed wavelength. The proposed configuration for broadband polarization conversion was also simulated to confirm its effectiveness in polarization state modulation. These results suggest the potential of a columnar thin film to be a perfect polarization modulator that can generate any polarization state over a wide range of wavelengths.

\section{References}

[1] Y. J. Jen and C. L. Chiang, "Enhanced polarization conversion for an anisotropic thin film," Opt. Commun. 265(2), 446-453 (2006) [doi:10.1016/j.optcom.2006.03.060].

[2] Y. J. Jen, C. W. Yu, C. F. Lin, Y. H. Liao, and C. Y. Peng, "Modulation of the polarization state of light using a weak anisotropic thin film," Opt. Lett. 33(5), 467469 (2008) [doi:10.1364/OL.33.000467].

[3] Y. J. Jen and C. Y. Peng, "Narrow-band and broad-band polarization conversion reflection filters," Appl. Phys. Lett. 89, 041128 (2006) [doi:10.1063/1.2243803].

[4] J. J. Steele and M. J. Brett, "Nanostructure engineering in porous columnar thin films: recent advances," J. Mater. Sci.: Mater. Electron. 18(4), 367-379 (2007) [doi:10.1007/s10854-006-9049-8].

[5] A. Lakhtakia and R. Messier, Sculptured Thin Films: Nanoengineered Morphology and Optics, SPIE Press, Bellingham, WA (2005).

[6] I. J. Hodgkinson and Q. H. Wu, Birefringent Thin Films and Polarizing Elements, World Scientific, Singapore (1997).

[7] R. M. A. Azzam and N.M. Bashara, Ellipsometry and Polarized Light, pp. 6-51, Elsevier, Amsterdam, North-Holland (1986).

[8] Y. J. Jen, C. Y. Peng, and H. H. Chang, "Optical constant determination of an anisotropic thin film via polarization conversion," Opt. Exp. 15(8), 4445-4451 (2007) [doi:10.1364/OE.15.004445]. 